\title{
Efficiency Assessment of Wind Farms: a Two-stage Approach
}

\author{
C. B. Vaz* and A. P. Ferreira ${ }^{\dagger}$ \\ *Polytechnic Institute of Bragança, Campus de Santa Apolónia, Apartado 1134, 5301-857, Portugal; \\ clvaz@ipb.pt; CGEI, Centre for Management and Industrial Engineering, INESC TEC \\ ${ }^{\dagger}$ Polytechnic Institute of Bragança, Campus de Santa Apolónia, Apartado 1134, 5301-857, Portugal; \\ apf@ipb.pt; CISE, Electromechatronic Systems Research Center, University of Beira Interior
}

\begin{abstract}
This study proposes an integrated methodology to provide insights regarding the efficiency of wind farms of two major promoters in the Portuguese energy sector. The focus of the wind farms performance assessment is on the operating stage which corresponds to the electrical energy production process. In a first stage, Data Envelopment Analysis is used to measure the efficiency of wind farms in producing electrical energy from the resources available and exogenous variables. This method is complemented by bootstrapping to refine the efficiency estimates. In a second stage, the factors which may influence the performance of wind farms are investigated by using truncated regression with bootstrapping. The methodology proposed may be used by promoters in management of wind farms and may also be useful to regulator agents on the supervision of the energy market.
\end{abstract}

Keywords: Data Envelopment Analysis; Efficiency; Bootstrap; Wind farm

PACS: $88.50 . j n$, 88.05.Jk, 88.05.Lg, 89.65.Gh

\section{INTRODUCTION}

In the last decades the wind generated electricity has grown significantly, since it is an alternative to fossil fuels and also environmental friendly. According to Global Wind Energy Council (GWEC), installed wind power capacity has grown to cumulative worldwide installation level of $318 \mathrm{GW}$, with $35.5 \mathrm{GW}$ installed in 2013. Portugal mainland accounts for about $4 \%$ of the wind energy installed capacity of the European Union, with approximately $4.8 \mathrm{GW}$ of accumulated installed capacity in 2013 which is capable to generate about $24 \%$ of the electrical energy demand [1]. Currently, there are more than 10 promoters exploring and managing the wind farms in Portugal. Thus, the performance assessment of wind farms can be crucial for promoters and decision makers, which are interested in identifying the farms that explore in a better way the resources available.

Data Envelopment Analysis (DEA) has been accepted as an important method for benchmarking energy sectors in many countries, particularly in the electricity sector [2], although its application in the wind sector is still scarce [3]. The development of wind energy sector and the growing need of performance assessment models for promoters and regulators motivated this study. For promoters, it is important to assess the performance of wind farms where the production is mainly depend on the wind availability. The performance assessment enables the identification of best practices and factors which influence farms efficiency. This information can be used to improve the performance of inefficient farms, and also to verify if the different technologies available and installed in each farm influence the results achieved. It can also be used to support projects of new wind farms and on repowering processes, contributing to decrease the investment costs. In the regulation context, the performance assessment of wind farms may be used in the distribution of new licenses, renewal of existing ones and promoting the farms that have the highest levels of performance. The robustness of the scores achieved by DEA models can be tested by using bootstrapping framework [4]. The identification of factors which may influence the performance of wind farms can be performed by using bootstrap truncated regression [5]. This study assesses the performance of wind farms managed by two promoters, during 2010 and 2011 years, in which the wind energy sector suffered a considerable decrease in electric energy produced.

This paper is organized as follows. Next section describes the methodology used to assess the performance of wind farms. The following section presents the case study in the wind energy sector, the results and discusses their implications. Finally, the last section rounds up the main conclusions. 


\section{PERFORMANCE ASSESSMENT METHODOLOGY}

In a first stage, DEA is used to assess the farms efficiency by taking into account the resources and the nondiscretionary variable, the wind, available in each farm to produce electric energy. In a second stage, potential factors that may explain the variations in efficiency scores among farms are explored by using bootstrap truncated regression [5]. These factors can be related with technological and managerial policies of farms that impact in their performance.

DEA is a non-parametric approach to assess the relative efficiency of a homogeneous set of Decision Making Units (DMUs) in producing multiple outputs from multiple inputs. This allows the identification of the "best practices DMUs" and their linear combination defines the frontier technology. By reference to this frontier, a single summary measure of efficiency is calculated for each DMU. In the original DEA model proposed by Charnes et al. [6], the efficiency score of each DMU is estimated by using the frontier technology characterized by Constant Returns to Scale (CRS). Consider an input vector $\mathbf{x}=\left(x_{1}, \ldots, x_{m}\right) \in R_{+}^{m}$ used to produce an output vector $\mathbf{y}=\left(y_{1}, \ldots, y_{s}\right) \in R_{+}^{s}$ in a technology involving $n$ production units (as production possibility set). For an output maximizing perspective, the relative efficiency of a $\mathrm{DMU}_{o}$ can be assessed using the linear programming model (1).

$$
\max \left\{h_{o} \mid x_{i o} \geq \sum_{j=1}^{n} \lambda_{j} x_{i j}, \quad i=1, \ldots, m ; h_{o} y_{r o} \leq \sum_{j=1}^{n} \lambda_{j} y_{r j}, \quad r=1, \ldots, s ; \lambda_{j} \geq 0 ; \quad \forall_{j, i, r}\right\}
$$

The optimal solution of model (1), $h_{o}^{*}$ corresponds to the factor by which the outputs of the $\mathrm{DMU}_{o}$ can be expanded given the current level of resources used. The efficiency score, $\theta_{o}$, of the $\mathrm{DMU}_{o}$ under evaluation is the inverse of $h_{o}^{*}$, i.e. $\theta_{o}=\frac{1}{h_{o}^{*}}$. The measure of efficiency, $\theta_{o}$, equals to one when the unit under assessment is efficient, whereas lower scores indicate the existence of inefficiencies. For the inefficient units there is evidence that it is possible to obtain higher levels of outputs with the same or lower levels of the inputs currently used. In the model proposed by Banker et al. [7], the efficiency score for each DMU is estimated by using the frontier technology characterized by Variable Returns to Scale (VRS) which can be calculated by including the constraint $\sum_{j=1}^{n} \lambda_{j}=1$ in model (1). To correct the DEA efficiency estimates for bias we use the bootstrapping method proposed by Simar and Wilson [4], which is appropriate for use with the DEA efficiency estimates, which range from 0 to 1 . This procedure was implemented using the statistical software R including the FEAR package [8]. Thus, we derive for each DMU a confidence interval for the $\theta_{o}$, the bias and the bias-corrected efficiency, $\hat{\theta}_{o}$. These scores are used to assess the wind farms performance.

The performance of wind farms may be influenced by factors not included in the first stage. Understanding how such factors can be related to efficiency of wind farms is important to support the definition of practices for future performance improvement. This analysis is performed by using a truncated regression with bootstrapping, formulated according to the algorithm \#2 proposed by Simar and Wilson [5] and has been used in several studies [9, 10]. The model uses the bias-corrected efficiency as the dependent variable, and as regressors variables related to technological, environmental and managerial features. Due to the availability of data from two periods, we used a panel data truncated model controlling for time effect. Thus, the impact of variable $z_{j t}$ on $\theta_{j t}$ of wind farm $j$, in year $t$, is assessed by the relationship $\theta_{j t}=\alpha_{o}+\eta_{t}+z_{j t} \beta+\varepsilon_{j t}$. Subscript $j$ represents the $j$ th farm $(j=1, \ldots, n)$ and subscript $t$ represents the time period $(t=1, \ldots, l) . \alpha_{o}$ is the intercept, $\eta_{t}$ is a vector of dummy variables for each year, $z_{j t}$ represents the regressors, $\beta$ corresponds to the vector of coefficients to be estimated and $\varepsilon_{j t}$ is the error term with a $N\left(0, \sigma_{\varepsilon}^{2}\right)$ distribution with a truncation at $\left(1-\alpha_{o}-\eta_{t}-Z_{j t} \beta\right)$. Note that $\theta_{j t}$ corresponds to the efficiency of wind farm $j$, in year $t$, estimated using model (1) and corrected by bootstrapping.

\section{PERFORMANCE ASSESSMENT OF WIND FARMS: CASE STUDY}

This section applies the proposed methodology to evaluate the performance of wind farms from two major promoters (EDP and Iberwind) in the Portuguese energy sector. DMUs under analysis are wind farms which are formed by a group of aerogenerators connected to the public grid. Wind farms can be considered homogenous as they result from similar set-up stages and use similar generation processes. The efficiency assessment is addressed to the operating stage although the farm performance is also linked to decisions taken prior the start up phase [3]. The output-oriented perspective is used, as the objective of the farms is to maximize the active electric energy delivered to the public grid. Thus, the electric energy production is considered as output which is not restrained by demand, as currently regulated. Relating to a production function, the electric energy generated is a function of the capital and fuel, which in turn define the input set. The capital factor is associated with the installed power, and the number of aerogenerators in each farm. The fuel factor is mainly related with the wind availability in each farm. The available wind power in each 
TABLE 1. Mean and standard deviation (SD) values for inputs and output of wind farms

\begin{tabular}{ccccccc|c}
\hline & & $\begin{array}{c}\text { Rotor } \\
\text { diameter (m) }\end{array}$ & $\begin{array}{c}\text { No of } \\
\text { aerogenerators }\end{array}$ & $\begin{array}{c}\text { Inputs } \\
\text { Installed } \\
\text { power (MW) }\end{array}$ & $\begin{array}{c}\text { Wind } \\
\text { speed (m/s) }\end{array}$ & $\begin{array}{c}\text { Wutput } \\
\text { Wours }\end{array}$ & $\begin{array}{c}\text { Electric } \\
\text { Energy (GWh) }\end{array}$ \\
\hline 2010 & Mean & 74.82 & 12.15 & 20.71 & 4.53 & 3344.65 & 50.47 \\
& SD & 13.31 & 6.56 & 14.37 & 1.64 & 1756.78 & 37.05 \\
2011 & Mean & 74.82 & 12.15 & 20.80 & 4.24 & 3127.09 & 46.64 \\
& SD & 13.31 & 6.56 & 14.39 & 1.32 & 1524.42 & 34.19 \\
\hline
\end{tabular}

TABLE 2. Results of original and bootstrapped efficiency estimates

\begin{tabular}{lcccc|cccc}
\hline & & \multicolumn{2}{c}{$\mathbf{2 0 1 0}$} & & & $\mathbf{2 0 1 1}$ & \\
& Orig. Eff. & Bias-corr. Eff. & Bias & St.dev. & Orig. Eff. & Bias-corr. Eff. & Bias & St.dev. \\
\hline Mean & 0.850 & 0.813 & 0.036 & 0.018 & 0.787 & 0.747 & 0.040 & 0.019 \\
SD & 0.131 & 0.130 & 0.027 & 0.020 & 0.140 & 0.131 & 0.031 & 0.024 \\
\hline
\end{tabular}

aerogenerator is given by $P=\frac{1}{2} \times A \times \rho \times v^{3}$, where $A$ is the swept area of the rotor, $\rho$ is the air density, and $v$ is the linear velocity of the wind. To transpose the fuel factor to the model, we use the rotor diameter, the annual average wind speed at each site and also the annual number of hours in which the wind velocity is between cut in and cut out typical wind speeds, henceforth named wind hours. It is assumed that during the operating phase, the maintenance service is scheduled within downtime periods of wind farms activity ${ }^{1}$.

Final data set considers 46 wind farms with an installed power which ranges from 10 to $80 \mathrm{MW}$. A panel data set regarding 2010 and 2011 years was collected from Annual Reports and Accounts from promoters. The 46 farms under analysis are located in several wind typical areas in Portugal. For each area, the wind data is collected from a meteorological data base throughout identification of the station which represents its wind profile, defined by the nearest meteorological station ${ }^{2}$. The descriptive measures concerning inputs and output under analysis are summarized in Table 1.

\section{RESULTS AND DISCUSSION}

Concerning the assessment of wind farms efficiency (first stage), the efficiency score for each farm in each year is estimated based on comparison with a pooled frontier corresponding to the best practices observed in the two years analysed. As the scale size affects the productivity of a DMU, the scale of wind farms activity is investigated by exploring the distance between CRS and VRS frontiers through the test based on bootstrap, as proposed by Simar and Wilson [11]. The results indicated there is statistical evidence that wind farms activity is characterized by CRS. Thus, model (1) is used to assess the original efficiency of wind farms which includes sources related with resources under-utilization and scale size. The bootstrap estimates were produced using 2000 samples according to [4]. Table 2 reports the summary results for the original efficiency scores, the bias-corrected efficiency scores, the bias, and the standard deviations for the wind farms under analysis, in each year analysed.

For each farm, as the estimated bias is much larger than the standard deviation, the bias-corrected efficiency estimates are preferred to the original estimates [4]. In 2010, the average bias-corrected efficiency of $81.3 \%$ indicates that the potential for electric energy produced would increase $22.9 \%$ (i.e. 1/0.813) by maintaining the current resources and wind availability in farms, if wind farms would perform as efficiently as its benchmarks. The bias-corrected efficiency decreased, on average, $7 \%$ in 2011 . Thus, the potential for electric energy produced could increase $33.9 \%$. Meteorological data shows that in 2011 there was a declining in wind potential which can partially explain some deterioration on farms efficiency. We consider that the benchmarks have bias-corrected efficiency equal or higher than $90 \%$, in both years, as observed in three wind farms. Their best practices should be identified in order to be emulated by inefficient units. These practices may be related to the use of more efficient aerogenerators, enhanced wind farm design and layout or better operation and maintenance schemes.

\footnotetext{
1 Data concerning maintenance and operation costs are confidential and, for this reason, they are not included in the model.

2 The panel data is limited to 2010 and 2011 due to meteorological data regarding wind is not available for other years.
} 
TABLE 3. Truncated regression results

\begin{tabular}{lccc}
\hline Variable & Coefficient & Standard Error & p-value \\
\hline Age & -0.006 & 0.006 & 0.313 \\
2011 year & -0.095 & 0.037 & 0.010 \\
Synchronous generator & 0.082 & 0.049 & 0.092 \\
Iberwind & -0.049 & 0.040 & 0.212 \\
\hline
\end{tabular}

In order to investigate other factors which can have impact on inefficiencies of wind farms (second stage), the bootstrap truncated regression [5] is performed considering the age of the wind farm, the technology of the conversion system (based on synchronous or asynchronous generators) and the promoter as regressors and bias-corrected efficiency score as the dependent variable. Those factors are chosen as they may reflect technological developments over time, different control strategies and managerial policies which can impact on farms efficiency. The technology of the conversion system and the promoter are categorical variables which should be included as dummy variables, requiring the comparison of a variable to its reference level. The technology installed in each farm uses the reference level conversion system based on asynchronous generator. The promoter reference level is EDP. Due to the use of a panel data truncated model, the year is also used as dummy variable to control the time effect (the reference level is 2010 year). Table 3 summarizes the results from panel data truncated model in terms of coefficients, standard errors and p-values. The truncated regression model is statistically significant ( $\chi^{2}$ test with p-value equal to 0.0074$)$, with a pseudo- $R^{2}$ equal to 0.15 . We confirm that farms decreased significantly the efficiency level in 2011 , as stated before. Apparently, the farm age decreases slightly the efficiency level, although this effect is not significant. Compared to the farms that use technology based on asynchronous generators, the farms equipped with synchronous generators increased slightly their efficiency, with p-value equals to $9.2 \%$. To enhance the robustness of these results the sample size should be increased. Compared to the farms of EDP, the farms of Iberwind decreased slightly the efficiency level, although this effect is not significant.

\section{CONCLUSIONS}

This study proposes a methodology to assess the efficiency of wind farms, by applying bootstrap procedures to obtain statistical inference on the estimated scores. In a first stage, the performance assessment of wind farms enables to benchmark the farms, to identify benchmark profiles and intervention areas in order to improve inefficient farms. The second stage allows the identification of factors concerning technological developments over time, different control strategies and managerial policies, not included in the first stage, which may influence the performance of wind farms. This methodology can be useful in project design and layout of new farms and also in repowering and overpowering processes. Regarding the operating stage of the farms analysed, three units are the benchmarks, whose best practices can be related with well-performing operations and maintenance programs. Further research should be conducted using a larger panel data set in order to analyse the impact of wind availability on the productivity of wind farms. The inclusion of variables concerning the operation and maintenance schemes should also be explored in future performance assessments of wind farms.

\section{REFERENCES}

1. e2p, "Parques Eólicos em Portugal," December, 2013, URL http: //e2p.inegi . up .pt/.

2. P. Zhou, B. W. Ang, and K. L. Poh, European Journal of Operational Research 189, 1-18 (2008).

3. G. Iglesias, P. Castellanos, and A. Seijas, Energy Economics 32, 1199-1208 (2010).

4. L. Simar, and P. W. Wilson, Management Science 44, 49-61 (1998).

5. L. Simar, and P. W. Wilson, Journal of Econometrics 136, 31-64 (2007).

6. A. Charnes, W. W. Cooper, and E. Rhodes, European Journal of Operational Research 2, 429-444 (1978).

7. R. D. Banker, A. Charnes, and W. W. Cooper, Management Science 30, 1078-1092 (1984).

8. P. W. Wilson, Socio-Economic Planning Sciences 42, 247 - 254 (2008).

9. I. M. Horta, A. S. Camanho, J. Johnes, and G. Johnes, Journal of Productivity Analysis 39, 89-99 (2012).

10. J. Odeck, Omega 37, 1007-1017 (2009).

11. L. Simar, and P. W. Wilson, European Journal of Operational Research 139, 115-132 (2002). 